\section{Generalizability of measures: A computer program for two- and three-facet designs}

\author{
ODED ERLICH and RICHARD SHAVELSON \\ University of California, \\ Los Angeles, California 90024
}

\section{Generalizability Theory}

Classical test theory (Gulliksen, 1950) has been shown to be limited in handling a wide variety of measurement problems (Cronbach, Gleser, Nanda, \& Rajaratnam, 1972). To overcome some of the problems, Cronbach et al. (1972) developed a theory of the generalizability of behavioral measurements. This theory does not rely upon the restricted assumptions of the classical test theory. Furthermore, it isolates more than one source of error variation affecting the measurement.

Generalizability theory distinguishes two types of studies: a G study and a D study. A G study is a pilot study carried out according to an elaborate design which includes all possible sources that may influence the generalizability of measurement. A D study collects data for the purpose of making decisions or drawing conclusions. One can choose the design for the D study from information provided in the $\mathrm{G}$ study, considering, of course, the D study's purposes and the cost of collecting information.

In contrast to the classical test theory, generalizability theory distinguishes between absolute decisionsdecisions made on an individual's absolute score (e.g., $80 \%$ correct constitutes the criterion on a written driver's examination)-and relative decisions-decisions based upon rank ordering of individuals (e.g., correlational studies). This distinction is used to determine which sources of error variability affect the measurement.

In generalizability theory the sources of error affecting the measurement (e.g., judges, observation occasions, items) are called facets (cf. factors in the analysis of variance). The conditions (cf. levels of a factor in the analysis of variance) representing these facets either constitute a random sample from the universe of conditions (a random facet) or exhaust the universe of conditions (a fixed facet). The persons on whom the measurements are taken are considered a random sample from a well-defined target population. A variability in the measurement due to a facet or an interaction among facets is defined as error variance, while the variability among the persons is defined as the universe score variance. An observed score variance is the sum of the universe score variance and the sampling error variances. The sampling error variance is defined as an average error variance where the average is taken over the number of conditions in which the source of error is sampled. The coefficient of generalizability (the counterpart of reliability coefficient) is defined as the ratio of universe score variance to observed score variance.

\section{The Program}

The purposes of this program are to perform a $G$ study analysis and to provide data on alternative designs for D studies.

The program performs analyses for the following $G$ study designs: (a) two-facet random model design; (b) like a, but with one random facet nested within the other random facet; (c) three-facet mixed model with two random facets and one fixed facet; (d) like c, but with one random facet nested within the other random facet.

Program Calculations. The program calculates: Step 1: estimates of variance components involved in the design; Step 2: an estimate of the sampling error variance for each component involved in the design; Step 3: an estimate of the total sampling error variance for decisions based on rank ordering of individuals (e.g., correlational studies); Step 4: an estimate of the total sampling error variance for decisions based on the individuals' absolute scores (e.g., written driver's examination); Step 5: an estimate of the observed score variance; and Step 6: an estimate of the coefficient of generalizability. The calculations in Steps 2 through 6 are done for six chosen combinations of number of levels to the two random facets involved (e.g., if the two random facets are raters and observation occasions, the combinations may be two raters and four occasions, three raters and three occasions, etc.). Step 7: Four optimal alternative combinations of number of levels of the two random facets for a prespecified minimum required magnitude of the coefficient of generalizability. These four calculations are done for four prespecified values of number of levels of one random facet and a prespecified maximum number of levels of the other random facet. (For example, in an observational study, the minimum acceptable level of generalizability might be .7; the four levels of one random facet might be one, two, three, and four raters, and a maximum of 10 occasions might be considered.)

Input. The program input includes three control cards which apply to all variables to be analyzed under the same design, and an additional two control cards for each variable analyzed. The control cards include the following information: Card 1: number of subjects, number of levels of each facet (4F3.0); Card 2: six combinations of number of levels of the two random facets (12F3.0); Card 3: minimum required coefficient of generalizability (F3.0); Card 4: variable title card column 1-80; Card 5:sums of squares for all sources of variation involved in the design ( $5 \mathrm{~F} 15.5)$. There is no limit on the number of variables to be analyzed. 
Output. The program provides the following output: (1) Summary table for each source of variability including: degrees of freedom, mean square, variance estimate, percent of the total variance accounted for by the variance estimate (absolute decision), and percent of the total variance accounted for by the variance estimate (relative decision).

(2) Summary table for the G study analysis. For each source of variability the table includes: a variance estimate and estimates of sampling error for the six chosen combinations of number of levels of the two random facets. Also, for each of the six combinations, the table provides: total sampling error variance and standard deviation for both absolute and relative decisions analyses, observed score variance and standard deviation, and coefficient of generalizability. (3) Plot of the coefficient of generalizability for: (a) the six chosen combinations of number of levels of the two random facets; (b) the four optimal alternative combinations of number of levels of the two random facets.

Computer and Language. IBM 360/91, WATFIV(FORTRAN); core requirement: 180K; execution time: less than $30 \mathrm{sec}$ for 100 variables.

Availability. The source listing of the program, its documentation, and a sample application may be obtained at no cost from Oded Erlich, UCLA Graduate School of Education, Los Angeles, California 90024.

\section{REFERENCES}

Cronbach, L. J., Gleser, G. C., Nanda, H., \& Rajaratnam, $\mathrm{N}$. The dependability of behavioral measurements: The theory of generalizability for scores and profiles. New York: Wiley, 1972.

Gulliksen, H. Theory of mental tests. New York: Wiley, 1950. 